Artículo Original/ Original Article

http://dx.doi.org/10.18004/mem.iics/1812-9528/2020.018.02.27

\title{
Evaluación de primers reportados para detección de KDR en especies de Anopheles colectadas en los departamentos de Caaguazú y Alto Paraná en Paraguay
}

\author{
*J onás Fernández ${ }^{1}$, Luis Ferreira ${ }^{2}$, Luciano Franco $^{2}$, Nidia Martínez $^{2},{ }^{*}$ Nilsa González $^{1}$, Ninfa \\ Vera de Bilbao ${ }^{3}$, Florencia del Puerto ${ }^{3}$ \\ ${ }^{1}$ Universidad Nacional de Asunción, Facultad de Ciencias Exactas y Naturales. San Lorenzo, Paraguay \\ ${ }^{2}$ Ministerio de Salud Pública y Bienestar Social, Servicio Nacional de Erradicación de Enfermedades \\ Transmitidas por Vectores (SENEPA), Departamento de Entomología. Asunción, Paraguay \\ ${ }^{3}$ Universidad Nacional de Asunción, Instituto de Investigaciones en Ciencias de la Salud, Departamento \\ de Medicina Tropical. San Lorenzo, Paraguay
}

Cómo referenciar este artículo/ How to reference this article:

\begin{abstract}
Fernández J, Ferreira L, Franco L, Martínez N, González N, Vera de Bilbao N, del Puerto F. Evaluación de primers reportados para detección de KDR en especies de Anopheles colectadas en los departamentos de Caaguazú y Alto Paraná en Paraguay. Mem. Inst. Investig. Cienc. Salud. 2020; 18(2): 27-32
\end{abstract}

\section{R E S U M E N}

Las mutaciones KDR en el gen del canal del sodio (VGSC) han sido ya detectadas en al menos 13 especies de mosquitos Anopheles en su mayoría especies de África, pero aún resta por determinar los cebadores específicos para la detección en especies de Latinoamérica. En nuestro país la especie Anopheles darlingi es el vector principal de la malaria, y el A. albitarsis, el vector secundario. Se emplearon muestras de mosquitos Anoheles de las especies A. strodei, A. albitarsis, A. fluminensis, A. evansae, A. nuneztovari, A. nyssorhynchela lutzi y A. oswaldoi capturadas en los departamentos de Caaguazú y Alto Paraná en Paraguay. Para la amplificación y secuenciación se usaron cebadores reportados para el gen VGSC de A. albimanus en Guatemala, que resultaron ser específicos solo para la especie A. strodei. La secuencia revela el codón TTA que codifica para una Leucina como la secuencia TTG, reportada para la versión susceptible en la posición L1014. El fragmento amplificado es de aproximadamente 225 pares de bases. A nuestro entender, esta es la primera caracterización del gen VGSC en mosquitos Anopheles del Paraguay y para la especie A. strodei.

Palabras clave: Anopheles, PCR, Canales de sodio dependientes de potencial (VGSC), polimorfismo KDR, A. strodei.

\section{Evaluation of primers reported for KDR detection in Anopheles species collected in the departments of Caaguazú and Alto Paraná in Paraguay}

\begin{abstract}
A B S T R A C T
KDR mutations in the sodium channel gene (VGSC) have already been detected in at least 13 species of Anopheles mosquitoes, mostly African species, but the molecular techniques for detection in Latin American species have yet to be determined. In our country, Anopheles darlingi species is the main vector of Malaria, and A. albitarsis, the secondary vector. We used samples of Anoheles from the species A. strodei, A. albitarsis, A. fluminensis, A. evansae, A. nuneztovari, A. nyssorhynchela lutzi and A.
\end{abstract}

* Estudiante de la Carrera de Biotecnología

Fecha de recepción: noviembre 2019. Fecha de aceptación: julio 2020

Autor correspondiente: Florencia del Puerto. Universidad Nacional de Asunción, Instituto de Investigaciones en Ciencias de la Salud, Departamento de Medicina Tropical. San Lorenzo, Paraguay Email: colepuerto@hotmail.com 
oswaldoi collected at the departments of Caaguazú and Alto Paraná in Paraguay. For the amplification and sequentiation, primers reported for the VGSC gen of A. strodei in Guatemala were used and were specific only for A. strode in this case. The sequence revealed the TTA codon that codifies for a leucine as the TTG sequence, reported for the susceptible version at position L1014. The amplified fragment is approximately 225 base pairs. To our knowledge, this is the first characterization of the VGSC gene in Anopheles mosquitoes in Paraguay and for the species A. strodei.

Keywords: Anopheles, PCR, Voltage-gated sodium channel (VGSC), KDR polymorphism, A. strode.

\section{NTRODUCCI ÓN}

La malaria es una enfermedad transmitida a los humanos a través de una picadura de un mosquito hembra del género Anopheles infectado con parásitos del género Plasmodium Se estima que, en 2010, hubo 216 millones de casos de malaria en el mundo y que esta enfermedad causó más de 150,000 muertes ${ }^{(1)}$.

Desde 2003, el control de la malaria se ha considerado exitoso, con solo 91 casos en 2009 y 23 casos entre enero y julio de 2010 (16 de ellos fueron casos autóctonos). Entre los casos, 94\% eran del departamento de Alto Paraná y $6 \%$ eran del departamento de Caaguazú(2).

Actualmente, el Servicio Nacional para la eliminación de la enfermedad (SENEPA) considera los departamentos de Alto Paraná y Caaguazú, que fueron regiones históricamente endémicas, como regiones con bajo riesgo de transmisión ${ }^{(2)}$.

La resistencia a insecticidas es uno de los ejemplos más interesantes de la rapidez con la que se dan los cambios a nivel evolutivo. El DDT y los piretroides son neurotoxinas que actúan sobre los canales de sodio dependientes de potencial modificando la cinética de su abertura, lo que resulta en una abertura prolongada de cada canal lo que conlleva a la parálisis y muerte del insecto. Uno de los mecanismos de resistencia a piretroides en insectos se conoce como resistencia knockdown (KDR en inglés) causado por la disminución de la sensibilidad en ese canal $^{(3)}$.

Los piretroides son los insecticidas más comúnmente usados para fumigación residual de interiores y el único en su clase, recomendado para la impregnación de mosquiteros debido a su relativa baja toxicidad para los mamíferos, pero con capacidad de producir efectos knockdown en insectos ${ }^{(4)}$.

Se ha investigado sobre la variabilidad del ADN en el gen del canal de sodio dependiente del potencial, comúnmente referido como gen VGSC (siglas en ingles por voltaje gatedsodium cannel), que codifica los segmentos 5 y 6 del dominio II transmembranal de la proteína, de manera a estimar la evolución de la resistencia a piretroides en poblaciones de manzanas a nivel mundial. La variabilidad del ADN en 38 secuencias, revelaron la existencia de una mutación KDR única (L1014F), involucrada en la resistencia a piretroides y se encuentra en esta región mencionada, la cual es el resultado de varias sustituciones convergentes ${ }^{(5)}$.

El fenotipo de Resistencia en el gen VGSC es comúnmente referido por una mutación particular $(\mathrm{L} 1014 \mathrm{~F} / \mathrm{S} / \mathrm{H})$ en el segmento $I I S 6^{(6,7)}$. Otras mutaciones en diferentes regiones del gen también confieren resistencia del tipo knockdown en algunos insectos ${ }^{(6,8)}$, pero entre los anophelinos este es el único locus donde las mutaciones han sido reportadas hasta ahora y que confieren resistencia ${ }^{(9-15)}$.

La presencia de la mutación, así como también sus efectos, han sido plenamente estudiadas en mosquitos anopheles. Hasta ahora, las mutaciones KDR han sido ya detectadas en al menos 13 especies (Anopheles gambiae, Anopheles arabiensis, Anopheles sinensis, Anopheles stephensi, Anopheles subpictus, Anopheles sacharovi, Anopheles culicifacies, Anopheles sundaicus, Anopheles aconitus, Anopheles vagus, Anopheles paraliae, Anopheles peditaeniatus and Anopheles albimanus) de poblaciones africanas, asiáticas y recientemente del continente americano. Se han descrito siete variantes de la mutación (L1014F, L1014S, L1014C, L1014W, N1013S, N1575Y y V1010L) pero con una alta prevalencia de la mutación L1014F ${ }^{(16)}$. 
El objetivo del presente trabajo consistió en detectar y caracterizar mutaciones KDR por técnicas moleculares en vectores anophelinos capturados en localidades históricamente endémicas bajo vigilancia epidemiológica.

\section{MATERI ALES Y MÉTODOS}

Se realizó un estudio observacional descriptivo de corte transversal empleando muestras de mosquitos Anoheles de las especies A. strodei, A. albitarsis, A. fluminensis, A. evansae, A. nuneztovari, A. nyssorhynchela lutzi y A. oswaldoi capturados en criaderos naturales, en los departamentos de Caaguazú y Alto Paraná en Paraguay, durante los meses de agosto, setiembre y noviembre de 2016 y diciembre de 2017. Estos meses se caracterizan por temperaturas cálidas y corresponden a la estación primaveral del país.

Los estudios fueron realizados en pooles ya que estas muestras se utilizaron previamente para otro proyecto y ya quedó el material genético guardado a -20 grados. Muestras disponibles: A. strodei (17 pooles), A. albitarsis (4), A. fluminensis(1), A. evansae(1), A. nuneztovari(1), A. nyssorhynchela lutzi(1) y A. oswaldoi(1). Número máximo de mosquito por pool fue de 10 y mínimo de 3.

Los pooles de mosquitos fueron sometidos a extracción de ADN empleando el kit Nucleo Spin ${ }^{\circledR}$ DNA Insect de Macherey-Nagel GmbH\& Co siguiendo el protocolo del mismo previo almacenamiento.

Para la identificación de la mutación KDR se empleó la técnica PCR anidada, siguiendo el protocolo propuesto por Lol J Cet al 2013. Empleando los cebadores AAKDRR ' (5 -GCAANGCTAAGAANAGRTTNAG-'3); AAKDRF (5'AGATGGAAYTTYACNGAYTTC-'3) y AAKDRF2 (5'-CATTCATTTATGATTGTGTTTCGTG'3)(17).La amplificación del segmento 6 del dominio II del gen VGSC empleando cebadores reportados se llevó a cabo en una mezcla de reacción de $25 \mu \mathrm{L}$ que contenía 17.55 $\mu \mathrm{L}$ de agua. $2.5 \mu \mathrm{L}$ de Buffer (10x), $2 \mu \mathrm{L} \mathrm{MgCl}_{2}(25 \mathrm{x}), 0.5 \mu \mathrm{L}$ de dN TPs, $0.1 \mu \mathrm{L}$ de cada cebador (AAKDRR y AAKDRF), $0.25 \mu \mathrm{L}$ de Polimerasa Hot Start Taq (Thermo scientific) y $2 \mu \mathrm{L}$ de ADN. Las condiciones de PCR consistieron en una desnaturalización inicial a 95ㅇ C por 3 min., seguido de 35 ciclos de 95ㅇ C por $45 \mathrm{sec}$., 40.5 o $\mathrm{C}$ por $45 \mathrm{sec}$ y $72^{\circ} \mathrm{C}$ por $1 \mathrm{~min}$. con una elongación final a $72^{\circ} \mathrm{C}$ por $5 \mathrm{~min}$ en un termociclador Simpli Amp ${ }^{\mathrm{TM}}$ Thermo Fisher Scientific. Se realizó una segunda PCR empleando los cebadores AAKDRR y AAKDRF2 utilizando las mismas especificaciones de reacción que en la primera PCR y como ADN molde el producto de la primera PCR. Las condiciones de la segunda PCR consistieron en una desnaturalización inicial a 950 C por 3 min., seguido de 40 ciclos de 95 o C por $45 \mathrm{sec}$, 51.5 o C por $45 \mathrm{sec}$ y 72 으 $\mathrm{C}$ por $1 \mathrm{~min}$. con una elongación final a $72^{\circ} \mathrm{C}$ por $5 \mathrm{~min}$ en un termociclador Simpli Amp ${ }^{\mathrm{TM}}$ Thermo Fisher Scientific.

La muestra fue secuenciada empleando el servicio de secuenciación de Macrogen ${ }^{\circledR}$ y la secuencia fue analizada empleando el software en línea Clustal Omega ${ }^{\circledR}$

\section{RESULTADOS}

\section{Amplificación de KDR}

Todos los pooles de cada especie fueron analizados a excepción de los pooles de A. strodei que se analizaron 9 de 17.

Los cebadores empleados fueron específicos para un alelo de kdr en el segmento 6 del dominio II del gen VGSC en mosquitos de la especie Anopheles strodei, no así para las demás especies de Anopheles empleadas para el ensayo en la cual no hubo amplificación.

\section{Secuenciación}

Los resultados de la secuenciación de una muestra de pool de la especie An. Strodei confirmó la amplificación del segmento del gen VGSC, con la presencia del tipo susceptible (wild type) TTA (L1014) (Figura 1). 
PY-Strodei CO-Albimanus

CR-Albimanus MX-Albimanus
ACCATTCTTCTTAGCAACTGTAGTTATAGgAaACTAafTCGTAAgtGCATTAAATGATGC 120 ACCATTCTTCTTAGCAACTGTAGTTATAGGAACTTGGTCGTAAGTG - . . . . . 50 ACCATTCTTCTTAGCAACTGTAGTTATAGGAAACTTCGTCGTAAGTG $\ldots$ ACCATTCTTCTTAGCAACTGTAGTTATAGGAACTGTGTCGTAAGTG - . - . - . - 50

Figura 1: Alineación del segmento del gen VGSC de An. strodeide Paraguay con An. albimanus de América latina (Colombia susceptible TTG L1014, Costa Rica resistente TTC 1014F y Mexico resistente TGT 1014C). El sitio polimórfico (posición aminoacídica 1014) está encerrado por un cuadro rojo.

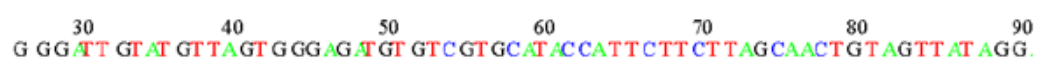
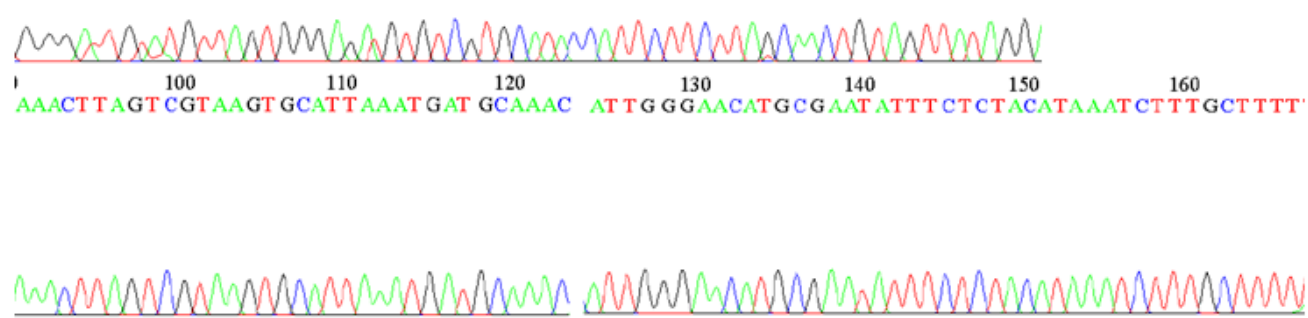

Figura 2: Electroferograma del segmento 6 del dominio II del gen VGSC de An. strodei.

Los picos en el electroferograma son únicos para el sitio de interés analizado, lo que demuestra la uniformidad del polimorfismo observado en cada mosquito del pool (Figura 2).

\section{DISCUSIÓN}

La caracterización de las mutaciones KDR en especies anophelinas de Latinoamérica todavía es bastante difícil debido a que no existen secuencias del gen VGSC completas o solo existen secuencias parciales disponibles en línea. La mayoría de las secuencias disponibles son de mosquitos de interés vectorial para la transmisión de la Malaria en África.

Los primers reportados para A. albimanus de Guatemala han demostrado ser específicos para amplificar ADN de A. strodei de Paraguay ${ }^{(17)}$. Si bien con esto pudimos obtener el producto de PCR del gen VGSC como para secuenciar y observar la versión susceptible del polimorfismo L1014, aun debemos colectar más mosquitos de la misma especie y realizar el monitoreo para conocer la frecuencia de es este polimorfismo. Es importante tener en cuenta que el Anopheles darlingi realmente es el que está considerado como el vector primario y el Anopheles albitarsis como vector secundario en nuestro país. En este contexto, las muestras empleadas en este estudio provienen de un estudio anterior donde la colecta se realizó en regiones históricamente endémicas de Paraguay (Caaguazú y Alto Paraná) y donde se colecto gran cantidad de la especie A. strodei en comparación con las otras especies anophelinas estudiadas, A. albitarsis, A. fluminensis, A. evansae, A nuneztovari, A.nyssorhynchela lutzi y A.oswaldoilo que podría ser de interés para la vigilancia entomológica.

Muy pocas especies de anopheles de Latinoamérica han sido caracterizadas en su polimorfismo L1014 para el gen VGSC, por tanto, existe una carencia de secuencias disponibles o métodos moleculares que permitan estudiar a los mosquitos Anopheles locales, constituye por tanto, un desafío establecer la técnica de PCR con los cebadores apropiados.

La secuenciación de la especie Anopheles strodei mostró que el pool analizado contenía el alelo kdr susceptible (wild type) TTA (L1014), previamente reportado en An. sacharovi, An. sinensis, An. albimanus y otras especies de Anopheles $\left({ }^{16,17,18,19,20)}\right.$. En otras poblaciones de mosquitos en Latinoamérica se ha detectado una mutación 
homocigota no sinónima, TGT (cisteína, L1014C), en muestras de campo de México. Esta mutación está asociada con la resistencia a permetrina, deltametrina y betacipermetrina en Ansinensis ${ }^{(16,21,22)}$. También se ha reportado en una muestra de Costa Rica contenía un polimorfismo TTC homocigota (fenilalanina, 1014L), esta mutación se asocia a la resistencia a permetrina y DDT en An. gambiae, resistencia deltametrina en Ansinensisy resistencia a DDT, permetrina, alfa-cipermetrina, lambda-cihalotrina y etofenprox en Anpeditaeniatus ${ }^{(9,19,22)}$.

Si bien analizamos 9 de 17 pooles de A. strodei porque consideramos suficiente para probar que los cebadores eran específicos, desde el punto de vista entomológico consideramos importante mencionar la existencia de los 17 pooles de dicha especie porque corresponden a un estudio previo, donde se puede observar que de la colecta realizada en departamentos históricamente endémicos, la especie mayormente colectada es el A. strodei, y podría constituirse en un potencial vector secundario de interés.

Hasta donde sabemos, ésta es la primera caracterización del gen VGSC en mosquitos Anopheles del Paraguay y para la especie A. strodei. Disponer de técnicas moleculares que permitan amplificar este gen, es importante para el monitoreo de sitios polimórficos que están asociados a conferir resistencia a insecticidas y sería un apoyo importante en la vigilancia para mantener al país libre de Malaria.

Declaración de conflicto de intereses: Los autores declaran que no existe conflicto de intereses.

\section{REFERENCI AS BIBLIOGRAFICAS}

1. Organización Mundial de la Salud. Resumen del Informe mundial 2012 sobre el paludismo [Internet]. 2012 [cited 2019 Nov 20]. Available from: http://www. who.int/malaria/publications/wo rld malaria_report_2012/en/index.html

2. Organizāción Panamericana de la Salud / Organización Mundial de la Salud (OPS / OMS). Manual de Diagnóstico microscópico de la Malaria. Programa Nacional de Control del Paludismo. SENEPA-MSP y BS. Patrocinado por el OPS / OMS; 2011.

3. Singh OP, Bali P, Hemingway J, Subbarao SK, Dash AP, Adak T. PCR-based methods for the detection of L1014 kdr mutation in Anopheles culicifacies sensu lato. Malar J [Internet]. 2009 Jul 14 [cited 2019 Oct 23];8:154. Available from: http:// www.ncbi.nlm.nih.gov/pubmed/ 19594 947. doi : 10.1186/1475-2875-8-154

4. World Health Organization. Global Malaria Programme \& World Health Organization. Malaria Unit. Indoor residual spraying : use of indoor residual spraying for scaling up global malaria control and elimination : WHO position statement. World Health Organization. 2006

5. Franck $P$, Siegwart $M$, Olivares J, Toubon $J-F$, Lavigne $C$. Multiple origins of the sodium channel $\mathrm{kdr}$ mutations in codling moth populations. PLoS One [Internet]. 2012 [cited 2019 Oct 23]; 7(8): e43543. Available from:

http://www.ncbi.nlm.nih.gov/pubmed/22912 889. doi.org/10.1371/journal. pone. 0043543 6. Soderlund DM, Knipple DC. The molecular biology of knockdown resistance to pyrethroid insecticides. Insect Biochem Mol Biol [Internet]. 2003 Jun [cited 2019 Oct 23];33(6):563-77. Available from: http://www.ncbi.nlm.nih.gov/ pubmed/ 12770 575. doi: 10.1016/s0965-1748(03)00023-7. 7. Davies TGE, Field LM, Usherwood PNR, Williamson MS. A comparative study of voltage-gated sodium channels in the Insecta: implications for pyrethroid resistance in Anopheline and other Neopteran species. Insect MolBiol [Internet]. 2007 J un [cited 2019 Oct 23]; 16(3): 361- 75. Available from: http://www.ncbi.nlm.nih.gov/pubmed/17433 $068 . \quad$ doi: 10.1111/j.13652583.2007.00733.x.

8. Kim H, Baek JH, Lee WJ, Lee SH. Frequency detection of pyrethroid resistance allele in Anopheles sinensis populations by real-time PCR amplification of specific allele (rtPASA). Pestic Biochem Physiol. 2007 Jan; 87(1): 54-61.

doi.org/10.1016/j.pestbp. 2006.06.009

9. Martinez-Torres D, Chandre F, Williamson MS, Darriet F, Berge JB, Devonshire AL, et al. Molecular characterization of pyrethroid knockdown resistance $(\mathrm{kdr})$ in the major malaria vector Anopheles gambiaes. S. Insect Mol Biol 1998, 7:179-184. doi: 10.1046/j.1365-2583. 1998.72062.x

10. Ranson $H$, Jensen $B$, Vulule J M, Wang $X$, Hemingway $\mathrm{J}$, Collins $\mathrm{FH}$. Identification of a point mutation in the voltage-gated sodium channel gene of Kenyan Anopheles gambiae associated with resistance to DDT and pyrethroids. Insect Mol Biol 2000, 9:491-7. doi: 10.1046/j.1365-2583.2000.00209.x. 
11. Syafruddin D, Hidayati AP, Asih PB, Hawley WA, Sukowati S, Lobo NF. Detection of $1014 \mathrm{~F} \mathrm{kdr}$ mutation in four major Anopheline malaria vectors in Indonesia. Malar J 2010, 9:315. doi.org/10.1186/14752875-9-315

12. Enayati $A A$, Vatandoost $H$, Ladonni $H$, Townson H, Hemingway J. Molecular evidence for a kdr-like pyrethroid resistance mechanism in the malaria vector mosquito Anopheles stephensi. Med Vet Entomol 2003, 17:138-144. doi: 10.1046/j.13652915.2003.00418.x.

13. Singh OP, Dykes CL, Das MK, Pradhan S, Bhatt RM, Agrawal OP, Adak T:

Presence of two alternative kdr-like mutations, L1014F and L1014S, and a novel mutation, V1010L, in the voltage gated $\mathrm{Na}+$ channel of Anopheles culicifacies from Orissa. India. Malar J 2010, 9:146. doi: 10.1186/1475-2875-9-146.

14. Singh OP, Dykes CL, Lather M, Agrawal OP, Adak T: Knockdown resistance

(kdr)-like mutations in the voltage-gated sodium channel of a malaria vector Anopheles stephensi and PCR assays for their detection. Malar J. 2011, 10:59. doi.org/10.1186/1475-2875-10-59

15. Kim $\mathrm{H}$, Baek JH, Lee $\mathrm{W}$, Lee $\mathrm{SH}$ : Frequency detection of pyrethroid resistance allele in Anopheles sinensis populations by real-time PCR amplification of specific allele (rtPASA). PesticBiochemPhysiol 2007, 87:54-61. doi.org/10.1016/S12268615(08)60317-9

16. Silva APB, Santos JMM, Martins AJ . Mutations in the voltage-gated sodium channel gene of anophelines and their association with resistance to pyrethroids - a review. Parasit Vectors [Internet]. 2014 Oct 7 [cited 2019 Oct 23]; 7:450. Available from: http://www.ncbi.nlm.nih.gov/pubmed/25292 318. doi: 10.1186/1756-3305-7-450.

17. Lol JC, Castellanos ME, Liebman KA, Lenhart $A$, Pennington PM, Padilla NR. Molecular evidence for historical presence of knock-down resistance in Anopheles albimanus, a key malaria vector in Latin America. Parasit Vectors [Internet]. 2013 Sep 18 [ cited 2019 Oct 22];6:268. Available from:

http://www.ncbi.nlm.nih.gov/pubmed/24330 978. doi: 10.1186/1756-3305-6-268.

18. Lüleyap HU, Alptekin D, Kasap H, Kasap M. Detection of knockdown resistance mutations in Anopheles sacharovi (Diptera: Culicidae) and genetic distance with Anopheles gambiae (Diptera: Culicidae) using CDNA sequencing of the voltage-gated sodium channel gene. J Med Entomol [Internet]. 2002 Nov [cited 2019 Oct 23];39(6):870-4. Available from: http://www.ncbi.nlm.nih.gov/pubmed/12495 185. doi: 10.1603/0022-2585-39.6.870

19. Verhaeghen K, Van Bortel W, Trung HD, Sochantha T, Keokenchanh K, Coosemans M. Knockdown resistance in Anopheles vagus, An. sinensis, An. paraliae and An. peditaeniatus populations of the Mekong region. Parasit Vectors [Internet]. $2010 \mathrm{Jul}$ 21 [cited 2019 Oct 23];3(1):59. Available from:

http://www.ncbi.nlm.nih.gov/ pubmed/20646 327. doi: 10.1186/1756-3305-3-59

20. Syafruddin D, Hidayati AP, Asih PB, Hawley WA, Sukowati S, Lobo NF. Detection of $1014 \mathrm{~F} \mathrm{kdr} \mathrm{mutation} \mathrm{in} \mathrm{four} \mathrm{major}$ Anopheline malaria vectors in Indonesia. Malar J. 2010; 9(1). doi.org/10.1186/14752875-9-315

21. Tan W-L, Wang Z-M, Li C-X, Chu H-L, Xu $Y$, Dong $Y-D$, et al. First report on cooccurrence knockdown resistance mutations and susceptibility to beta-cypermethrin in Anopheles sinensis from Jiangsu Province, China. PLoS One [Internet]. 2012 [cited 2019 Oct 23]; 7(1): e29242. Available from: http://www.ncbi.nlm.nih.gov/pubmed/22272 229. doi.org/10.1371/journal. pone. 0029242 22. Zhong D, Chang X, Zhou G, He Z, Fu F, Yan $Z$, et al. Relationship between knockdown resistance, metabolic detoxification and organismal resistance to pyrethroids in Anopheles sinensis. PLoS One [Internet] 2013 [cited 2019 Oct 23]; 8(2):e55475. Available from: http://www.ncbi.nlm.nih.gov/ pubmed/23405 157. doi.org/10.1371/journal.pone. 0055475 